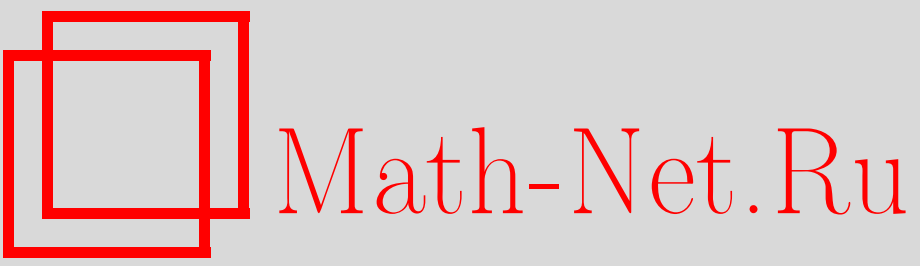

Л. С. Кузьменков, С. Г. Максимов, В. В. Федосеев, Микроскопическая квантовая гидродинамика систем фермионов. I, ТМФ, 2001, том 126, номер 1, 136-148

DOI: https://doi.org/10.4213/tmf420

Использование Общероссийского математического портала Math-Net.Ru подразумевает, что вы прочитали и согласны с пользовательским соглашением

http://www.mathnet.ru/rus/agreement

Параметры загрузки:

IP: 54.197 .130 .99

26 апреля 2023 г., 18:15:28 
ТЕОРЕТИЧЕСКАЯ

И МАТЕМАТИЧЕСКАЯ

ФИЗИКА

Том 126, № 1

январь, 2001

(C) 2001 г. Л.С. Кузьменков* , С.Г. Максимов* , В.В. Федосеев*

\section{МИКРОСКОПИЧЕСКАЯ КВАНТОВАЯ ГИДРОДИНАМИКА СИСТЕМ ФЕРМИОНОВ. I}

На основании уравнения Шредингера выведены фундаментальные уравнения микроскопической квантовой гидродинамики фермионов во внешнем электромагнитном поле (уравнения баланса частиц, импульса, энергии и магнитного момента). Произведено уточнение вида гамильтониана спин-спинового взаимодействия. Показано, что для многочастичной системы фермионов процедура замыкания системы уравнений баланса требует введения эффективного одночастичного уравнения Шредингера.

Коллективные физические процессы в системах взаимодействующих частиц связаны с перераспределением числа частищ, импульса, энергии, электромагнитного поля в физическом пространстве трех измерений. Явный учет причинно-следственных связей в динамике таких процессов приводит к необходимости перехода от описания системы частиц при помощи волновой функции, заданной в конфигурационном пространстве, к описанию при помощи функций в физическом пространстве. Эта задача для частиц без собственного магнитного момента рассмотрена в работе [1], где показано, что уравнение Шредингера для системы многих частиц может быть представлено в виде, вообше говоря, бесконечной системы уравнений гидродинамического типа. Фундаментальными среди этих уравнений являются уравнения баланса числа частиц, баланса импульса частиц и поля, баланса энергии (уравнения микроскопической квантовой гидродинамики). В отличие от результатов работ [2] (см. с. 269-287) и [3], эти микроскопические уравнения получены лишш на основании квантово-механического определения вероятности. Вместе с тем широкий класс нестационарных задач в атомной физике, физике конденсированного состояния вешества и физике плазмы не может быть рассмотрен без эффектов, обусловленных собственными магнитными моментами частиц, которые дают вклад в уравнения баланса. Поэтому в предлагаемой работе дается вывод фундаментальных уравнений микроскопической квантовой гидродинамики для системы фермионов во внешнем электромагнитном поле, среди которых есть и уравнение баланса для плотности магнитного момента. Эта задача потребовала также уточнения вида гамильтониана спин-спинового взаимодействия. Так как фундаментальные уравнения микроскопической квантовой гидродинамики не образуют замкнутого математического аппарата, рассмотрена общая проблема замыкания уравнений баланса.

${ }^{*}$ Московский государственный университет, Москва, Россия. E-mail: 1sk@nabla.phys.msu.su, serguey@teor242.phys.msu.su, wladdy@teor242.phys.msu.su 
В части II данной работы выгислены корреляционные функции, обменная энергия спин-спинового взаимодействия на основании непротиворечивого гамильтониана спинспинового взаимодействия. В качестве приложения метода микроскопической квантовой гидродинамики рассмотрена задача о многоэлектронном атоме, для которой получена замкнутая система уравнений. Эта задача является обобщением известной модели Томаса-Ферми-Дирака на случай нестационарных состояний атома и учитьвает обменные спин-спиновые взаимодействия. Для стационарных состояний найден интеграл уравнения баланса импульса, который определяет связь между концентрацией электронов и потенциалом поля.

1. Гамильтониан спин-спинового взаимодействия. Взаимодействие собственных магнитных моментов частиц подобно взаимодействию магнитных диполей. Поэтому гамильтониан спин-спинового взаимодействия обычно принимается в виде [4]-[6]

$$
\mathcal{H}=\frac{1}{2} \sum_{i, j=1}^{N} \frac{1}{r_{i j}^{3}}\left(\delta^{\alpha \beta}-\frac{3 x_{i j}^{\alpha} x_{i j}^{\beta}}{r_{i j}^{2}}\right) M_{i}^{\alpha} M_{j}^{\beta}=-\frac{1}{2} \sum_{i, j=1}^{N} \frac{\partial^{2}}{\partial x_{i}^{\alpha} \partial x_{i}^{\beta}} \frac{1}{r_{i j}} M_{i}^{\alpha} M_{j}^{\beta},
$$

где штрих у знака суммы означает $i \neq j, \mathbf{r}_{i j}=\mathbf{r}_{i}-\mathbf{r}_{j}, x_{i j}^{\alpha}=x_{i}^{\alpha}-x_{j}^{\beta}, \quad \alpha=1,2,3$, а $i, j$ - номера частищ или ячеек кристалла. Такой гамильтониан приводит к отличной от нуля дивергенции магнитной индукции. При этом, однако, не учитывается тот факт, что дивергенция магнитной индукции равна нулю всюду. Действительно, магнитная индукция системы $N$ магнитных диполей в вакууме равна

$$
B^{\alpha}(\mathbf{r})=\sum_{j=1}^{N} \frac{\partial^{2}}{\partial x^{\alpha} \partial x^{\beta}} \frac{1}{\left|\mathbf{r}-\mathbf{r}_{j}\right|} M_{j}^{\beta}=\int d \mathbf{r}^{\prime} \frac{\partial^{2}}{\partial x^{\alpha} \partial x^{\beta}} \frac{1}{\left|\mathbf{r}-\mathbf{r}^{\prime}\right|} \sum_{j=1}^{N} M_{j}^{\beta} \delta\left(\mathbf{r}^{\prime}-\mathbf{r}_{j}\right),
$$

где $\sum_{j=1}^{N} \mathbf{M}_{j} \delta\left(\mathbf{r}^{\prime}-\mathbf{r}_{j}\right) \equiv \mathbf{M}\left(\mathbf{r}^{\prime}\right)$ - микроскопическая плотность магнитного момента. Простое дифференцирование соотношения (2) дает

$$
\operatorname{div} \mathbf{B}=\int d \mathbf{r}^{\prime} \frac{\partial}{\partial x^{\beta}} \Delta \frac{1}{\left|\mathbf{r}-\mathbf{r}^{\prime}\right|} M^{\beta}\left(\mathbf{r}^{\prime}\right)=-4 \pi \operatorname{div} \mathbf{M}(\mathbf{r})
$$

и приводит, таким образом, к отличным от нуля источникам магнитного поля. В этом случае, как легко видеть, $\operatorname{rot} \mathbf{B}=0$.

Корректный вид гамильтониана спин-спинового взаимодействия можно получить непосредственно из классических уравнений Максвелла

$$
\operatorname{div} \mathbf{B}=0, \quad \operatorname{rot}(\mathbf{B}-4 \pi \mathbf{M})=0
$$

где $\mathbf{M}(\mathbf{r}, t)$ - плотность собственных магнитных моментов электронов. Из уравнений (3) имеем

$$
\Delta B^{\alpha}=4 \pi\left(\delta^{\alpha \beta} \Delta-\frac{\partial^{2}}{\partial x^{\alpha} \partial x^{\beta}}\right) M^{\beta}
$$


Магнитную индукцию можно представить в виде

$$
B^{\alpha}(\mathbf{r})=\int d \mathbf{r}^{\prime} G^{\alpha \beta}\left(\mathbf{r}-\mathbf{r}^{\prime}\right) M^{\beta}\left(\mathbf{r}^{\prime}\right)
$$

Подставляя (5) в (4), находим уравнение для функции Грина $G^{\alpha \beta}(\mathbf{r})$ :

$$
\Delta G^{\alpha \beta}(\mathbf{r})=4 \pi\left(\delta^{\alpha \beta} \Delta-\frac{\partial^{2}}{\partial x^{\alpha} \partial x^{\beta}}\right) \delta(\mathbf{r})
$$

В фурье-представлении $G^{\alpha \beta}$ имеет вид

$$
G^{\alpha \beta}(\mathbf{k})=4 \pi\left(\delta^{\alpha \beta}-\frac{k^{\alpha} k^{\beta}}{\mathbf{k}^{2}}\right)
$$

поэтому

$$
G^{\alpha \beta}(\mathbf{r})=\frac{\partial^{2}}{\partial x^{\alpha} \partial x^{\beta}} \frac{1}{r}+4 \pi \delta^{\alpha \beta} \delta(\mathbf{r})
$$

На основании принципа суперпозиции искомый гамильтониан равен

$$
\mathcal{H}=-\frac{1}{2} \sum_{i, j=1}^{N} G_{i j}^{\alpha \beta} M_{i}^{\alpha} M_{j}^{\beta}, \quad G_{i j}^{\alpha \beta}=G^{\alpha \beta}\left(\mathbf{r}_{i}-\mathbf{r}_{j}\right)
$$

При переходе к квантово-механическому описанию функцию $M_{i}^{\alpha}$ следует заменить оператором $\widehat{M}_{i}^{\alpha}=\mu_{\mathrm{B}} \hat{\sigma}_{i}^{\alpha}$ магнитного момента $i$-й частицы.

Для двух фермионов гамильтониан спин-спинового взаимодействия может быть получен из релятивистского выражения для амплитуды рассеяния двух фермионов (см. с. $383-390$ в книге [7]):

$$
\begin{gathered}
M_{\mathrm{fi}}=e^{2}\left(\bar{u}_{1}^{\prime} \gamma^{0} u_{1}\right) D^{00}\left(p_{1}^{\prime}-p_{1}\right)\left(\bar{u}_{2}^{\prime} \gamma^{0} u_{2}\right)+e^{2}\left(\bar{u}_{1}^{\prime} \gamma^{\alpha} u_{1}\right) D^{\alpha \beta}\left(p_{1}^{\prime}-p_{1}\right)\left(\bar{u}_{2}^{\prime} \gamma^{\beta} u_{2}\right) \\
p_{1}+p_{2}=p_{1}^{\prime}+p_{2}^{\prime}, \quad \alpha, \beta=1,2,3
\end{gathered}
$$

с последующим разложением ее в ряд до членов $\sim 1 / c^{2}$ (известное уравнение Брейта). Здесь фотонный пропагатор в кулоновской калибровке имеет вид

$$
D^{00}=-\frac{4 \pi}{\mathbf{q}^{2}}, \quad D^{0 \alpha}=0, \quad D^{\alpha \beta}=-\frac{4 \pi}{\frac{\omega^{2}}{c^{2}}-\mathbf{q}^{2}}\left(\delta^{\alpha \beta}-\frac{q^{\alpha} q^{\beta}}{\mathbf{q}^{2}}\right) .
$$

Биспинорная амплитуда свободной частицы $u_{\mathbf{p}}$ с требуемой точностью выражается через спинорную амплитуду $w_{\mathbf{p}}$ волновой функции $\varphi$ :

$$
u_{\mathbf{p}}=\sqrt{2 m c^{2}}\left(\begin{array}{c}
\left(1-\frac{\mathbf{p}^{2}}{8 m^{2} c^{2}}\right) w_{\mathbf{p}} \\
\frac{(\boldsymbol{\sigma} \mathbf{p})}{2 m c} w_{\mathbf{p}}
\end{array}\right), \quad \varphi(\mathbf{r})=\sum_{\mathbf{p}} \frac{1}{\sqrt{2 \varepsilon V}} w_{\mathbf{p}} \exp (i \mathbf{p r})
$$


С точностью до членов $\sim 1 / c^{2}$ волновая функция $\varphi$ удовлетворяет уравнению Шредингера

$$
\left(\frac{\widehat{\mathbf{p}}^{2}}{2 m}-\frac{\widehat{\mathbf{p}}^{4}}{8 m^{3} c^{2}}\right) \varphi=\left(\varepsilon-m c^{2}\right) \varphi
$$

Из (10)-(12) получаем

$$
\begin{gathered}
M_{\mathrm{fi}}=-4 m_{1} m_{2} c^{4} w_{1}^{\prime+} w_{2}^{\prime+} \widehat{U}\left(\mathbf{p}_{1}, \mathbf{p}_{2}, \mathbf{q}\right) w_{1} w_{2}, \\
\widehat{U}\left(\mathbf{p}_{1}, \mathbf{p}_{2}, \mathbf{q}\right)=4 \pi e^{2}\left\{\frac{1}{\mathbf{q}^{2}}-\frac{1}{8 m_{1}^{2} c^{2}}-\frac{1}{8 m_{2}^{2} c^{2}}+\frac{\left(\mathbf{q p}_{1}\right)\left(\mathbf{q p}_{2}\right)}{m_{1} m_{2} c^{2} \mathbf{q}^{4}}-\frac{\left(\mathbf{p}_{1} \mathbf{p}_{2}\right)}{m_{1} m_{2} c^{2} \mathbf{q}^{2}}+\frac{i \hat{\boldsymbol{\sigma}}_{1}\left[\mathbf{q p}_{1}\right]}{4 m_{1}^{2} c^{2} \mathbf{q}^{2}}-\right. \\
\left.-\frac{i \hat{\boldsymbol{\sigma}}_{1}\left[\mathbf{q p}_{2}\right]}{2 m_{1} m_{2} c^{2} \mathbf{q}^{2}}-\frac{i \hat{\boldsymbol{\sigma}}_{2}\left[\mathbf{q}_{2}\right]}{4 m_{2}^{2} c^{2} \mathbf{q}^{2}}+\frac{i \hat{\boldsymbol{\sigma}}_{2}\left[\mathbf{q} \mathbf{p}_{1}\right]}{2 m_{1} m_{2} c^{2} \mathbf{q}^{2}}\right\}+\widehat{U}_{\mathrm{spin}}(\mathbf{q})
\end{gathered}
$$

где интересуюший нас потенциал спин-спинового взаимодействия имеет вид

$$
\widehat{U}_{\mathrm{spin}}(\mathbf{q})=\frac{e^{2}}{4 m_{1} m_{2} c^{2}} 4 \pi\left(\frac{q^{\alpha} q^{\beta}}{\mathbf{q}^{2}}-\delta^{\alpha \beta}\right) \hat{\sigma}_{1}^{\alpha} \hat{\sigma}_{2}^{\beta},
$$

а в координатном представлении

$$
\begin{aligned}
\widehat{U}_{\text {spin }}(\mathbf{r}) & =\frac{1}{(2 \pi)^{3}} \int d \mathbf{q} \widehat{U}_{\text {spin }}(\mathbf{q}) \exp (i \mathbf{q r})= \\
& =\frac{e^{2}}{4 m_{1} m_{2} c^{2}} \hat{\sigma}_{1}^{\alpha} \hat{\sigma}_{2}^{\beta}\left(-\frac{\partial^{2}}{\partial x^{\alpha} \partial x^{\beta}} \frac{1}{(2 \pi)^{3}} \int 4 \pi \frac{d \mathbf{q}}{\mathbf{q}^{2}} \exp (i \mathbf{q r})-4 \pi \delta^{\alpha \beta} \delta(\mathbf{r})\right)= \\
& =\frac{e^{2}}{4 m_{1} m_{2} c^{2}} \hat{\sigma}_{1}^{\alpha} \hat{\sigma}_{2}^{\beta}\left(-\frac{\partial^{2}}{\partial x^{\alpha} \partial x^{\beta}} \frac{1}{r}-4 \pi \delta^{\alpha \beta} \delta(\mathbf{r})\right) .
\end{aligned}
$$

Этот результат находится в согласии с найденным выше выражением для функции Грина (8). Следуюшим шагом в получении гамильтониана спин-спинового взаимодействия является выделение $\delta$-функции, присутствуюшей неявно в выражении $-\partial^{2}(1 / r) / \partial x^{\alpha} \partial x^{\beta}$, путем усреднения по всем направлениям вектора $\mathbf{r}$ (см. [7]):

$$
-\frac{\partial^{2}}{\partial x^{\alpha} \partial x^{\beta}} \frac{1}{r}=\frac{\delta^{\alpha \beta}}{r^{3}}-\frac{3 x^{\alpha} x^{\beta}}{r^{5}}+\frac{4 \pi}{3} \delta^{\alpha \beta} \delta(\mathbf{r}) .
$$

Действительно, взяв след от обеих частей равенства (15), получаем тождество. В результате спин-спиновая часть потенциала Брейта приобретает вид

$$
\widehat{U}_{\mathrm{spin}}(\mathbf{r})=\frac{e^{2}}{4 m_{1} m_{2} c^{2}} \hat{\sigma}_{1}^{\alpha} \hat{\sigma}_{2}^{\beta}\left(\frac{\delta^{\alpha \beta}}{r^{3}}-\frac{3 x^{\alpha} x^{\beta}}{r^{5}}-\frac{8 \pi}{3} \delta^{\alpha \beta} \delta(\mathbf{r})\right) .
$$

В теории ядерного магнитного резонанса вводится так называемое контактное взаимодействие Ферми $\left(-8 \pi \mu_{\mathrm{B}}^{2} \hat{\boldsymbol{\sigma}}^{2} \delta(\mathbf{r}) / 3\right)$ для устранения неопределенности типа $0 \times \infty$, возникающей при вычислении квантово-механического среднего от гамильтониана спинспинового взаимодействия в форме (1) (см. с. 95-102 в книге [8]). 
Однако, как и выражение (1), формула (16) приводит к отличной от нуля дивергенции магнитной индукции. Причина такого противоречия лежит в некорректном выделении $\delta$-функции в формуле (15). Несмотря на то что след от равенства (15) приводит к тождеству, дифференцирование левой части (15) по $x^{\beta}$ дает

$$
-\frac{\partial}{\partial x^{\alpha}} \Delta \frac{1}{r}=4 \pi \frac{\partial}{\partial x^{\alpha}} \delta(\mathbf{r})
$$

а для правой части (15) получаем

$$
\frac{4 \pi}{3} \frac{\partial}{\partial x^{\alpha}} \delta(\mathbf{r})
$$

По-видимому, в этом случае необходимы более тонкие методы анализа обобщенных функций (см. с. 320 в книге [9]). Здесь для нас важным является тот факт, что гамильтониан спин-спинового взаимодействия, свободный от указанных противоречий, должен быть принят в виде

$$
\mathcal{H}=-\frac{1}{2} \sum_{i, j=1}^{N} G_{i j}^{\alpha \beta} M_{i}^{\alpha} M_{j}^{\beta}, \quad G_{i j}^{\alpha \beta}=G^{\alpha \beta}\left(\mathbf{r}_{i}-\mathbf{r}_{j}\right) .
$$

Этот гамильтониан лежит в основе выводов, изложенных ниже.

2. Исходные уравнения. Рассмотрим находящуюся во внешнем электромагнитном поле систему $N$ взаимодействуюших фермионов с одинаковыми массами, зарядами и собственными магнитными моментами. Состояние системы $N$ фермионов задается волновой функцией в $3 N$-мерном конфигурационном пространстве, являющейся спинором $N$-го ранга:

$$
\psi_{S}(R, t)=\psi_{s_{1} \ldots s_{N}}\left(\mathbf{r}_{1}, \ldots, \mathbf{r}_{N}, t\right) .
$$

Динамика такой системы описывается уравнением Шредингера

$$
i \hbar \frac{\partial \psi_{S}(R, t)}{\partial t}=(\widehat{H} \psi)_{S}(R, t)
$$

Гамильтониан системы является матричной недиагональной формой по дискретным спиновым индексам, так что действие его на волновую функцию будет иметь вид

$$
(\widehat{H} \psi)_{S}(R, t)=\sum_{S^{\prime}} \widehat{H}_{S S^{\prime}} \psi_{S^{\prime}}(R, t)=\sum_{s_{1}^{\prime}, \ldots, s_{N}^{\prime}} \widehat{H}_{s_{1} \ldots s_{N} s_{1}^{\prime} \ldots s_{N}^{\prime}} \psi_{s_{1}^{\prime} \ldots s_{N}^{\prime}}(R, t) .
$$

Гамильтониан рассматриваемой системы равен

$$
\widehat{H}=\sum_{i=1}^{N}\left\{\frac{\widehat{\mathbf{D}}_{i}^{2}}{2 m}+e \varphi\left(\mathbf{r}_{i}, t\right)-\mu_{\mathrm{B}}\left(\hat{\boldsymbol{\sigma}}_{i} \mathbf{B}\left(\mathbf{r}_{i}, t\right)\right)\right\}+\frac{1}{2} \sum_{i, j=1}^{N}\left(e^{2} G_{i j}-\mu_{\mathrm{B}}^{2} G_{i j}^{\alpha \beta} \hat{\sigma}_{i}^{\alpha} \hat{\sigma}_{j}^{\beta}\right),
$$

где $\widehat{D}_{i}^{\alpha}=-i \hbar \nabla_{i}^{\alpha}-e A_{i}^{\alpha} / c, \mu_{\mathrm{B}}=e \hbar / 2 m c-$ магнетон Бора, $A_{i}^{\alpha} \equiv A^{\alpha}\left(\mathbf{r}_{i}, t\right)$ - векторный потенциал поля $\mathbf{B}(\mathbf{r}, t), G_{i j}=1 /\left|\mathbf{r}_{i}-\mathbf{r}_{j}\right|$ - функция Грина оператора Лапласа, а $G_{i j}^{\alpha \beta}$ 
определена формулами (8), (9). Для матриц Паули $\hat{\sigma}_{i}^{\alpha}$ справедливы коммутационные соотношения

$$
\left[\hat{\sigma}_{i}^{\alpha}, \hat{\sigma}_{j}^{\beta}\right]=2 i \delta_{i j} \varepsilon^{\alpha \beta \gamma} \hat{\sigma}_{i}^{\gamma},
$$

где суммирование производится только по повторяюшимся греческим индексам. Оператор $\hat{\sigma}_{i}^{\alpha}$ действует лиш на соответствуюшую спиновую координату спинора $\psi_{S}(R, t)$, т.е. его матричные элементы имеют вид

$$
\left(\hat{\sigma}_{i}^{\alpha}\right)_{S S^{\prime}} \equiv\left\langle S\left|\hat{\sigma}_{i}^{\alpha}\right| S^{\prime}\right\rangle=\hat{\sigma}_{s_{i} s_{i}^{\prime}}^{\alpha} \prod_{\substack{j=1 \\ j \neq i}}^{N} \delta\left(s_{j}-s_{j}^{\prime}\right)
$$

где $\hat{\sigma}_{s_{i} s_{i}^{\prime}}^{\alpha}$ - обычные матрицы Паули в пространстве $S U(2)$. Для перехода из конфигурационного пространства в физическое следует определить операторы материальных полей. Достаточно задать оператор плотности числа частиц (или концентрации) [1]. Тогда операторы плотностей потоков появляются автоматически в уравнениях непрерывности и баланса импульса.

Усредненная по квантово-механическим состояниям концентрация частиц есть величина

$$
\begin{aligned}
n(\mathbf{r}, t) & =\sum_{S} \int d R \psi_{S}^{*}(R, t) \hat{n}(\mathbf{r}, R) \psi_{S}(R, t)= \\
& =\sum_{S} \int d R \sum_{i=1}^{N} \delta\left(\mathbf{r}-\mathbf{r}_{i}\right) \psi_{S}^{*}(R, t) \psi_{S}(R, t)
\end{aligned}
$$

где $d R=\prod_{k=1}^{N} d \mathbf{r}_{k}, S=\left\{s_{1}, s_{2}, \ldots, s_{N}\right\}$.

3. Фундаментальные уравнения квантовой гидродинамики фермионов. Вывод уравнений квантовой гидродинамики со спином аналогичен приведенному в работе [1] с той лишь особенностью, что в данном случае следует воспользоваться коммутационными соотношениями (20). В результате уравнение непрерывности и уравнение баланса импульса приобретают вид

$$
\begin{gathered}
\frac{\partial n(\mathbf{r}, t)}{\partial t}+\frac{1}{m} \nabla^{\alpha} j^{\alpha}(\mathbf{r}, t)=0, \\
\frac{\partial j^{\alpha}(\mathbf{r}, t)}{\partial t}+\nabla^{\beta} \Pi^{\alpha \beta}(\mathbf{r}, t)=e n(\mathbf{r}, t) E_{\mathrm{ext}}^{\alpha}(\mathbf{r}, t)- \\
-\int d \mathbf{r}^{\prime} e^{2} \nabla^{\alpha} G\left(\mathbf{r}-\mathbf{r}^{\prime}\right) n_{2}\left(\mathbf{r}, \mathbf{r}^{\prime}, t\right)+\frac{1}{m} \frac{e}{c} \varepsilon^{\alpha \beta \gamma} j^{\beta}(\mathbf{r}, t) B_{\mathrm{ext}}^{\gamma}(\mathbf{r}, t)+ \\
+M^{\beta}(\mathbf{r}, t) \nabla^{\alpha} B_{\mathrm{ext}}^{\beta}(\mathbf{r}, t)+\int d \mathbf{r}^{\prime} \nabla^{\alpha} G^{\beta \gamma}\left(\mathbf{r}-\mathbf{r}^{\prime}\right) M^{\beta \gamma}\left(\mathbf{r}, \mathbf{r}^{\prime}, t\right),
\end{gathered}
$$

где определения плотности импульса частищ

$$
j^{\alpha}(\mathbf{r}, t)=\sum_{S} \int d R \sum_{i=1}^{N} \delta\left(\mathbf{r}-\mathbf{r}_{i}\right) \frac{1}{2}\left\{\psi_{S}^{*}\left(D_{i}^{\alpha} \psi_{S}\right)+\left(D_{i}^{\alpha} \psi_{S}\right)^{*} \psi_{S}\right\}
$$


и тензора плотности потока импульса

$$
\begin{aligned}
\Pi^{\alpha \beta}(\mathbf{r}, t)= & \sum_{S} \int d R \sum_{i=1}^{N} \delta\left(\mathbf{r}-\mathbf{r}_{i}\right) \frac{1}{4 m} \times \\
& \times\left\{\left(D_{i}^{\beta} \psi_{S}\right)^{*}\left(D_{i}^{\alpha} \psi_{S}\right)+\psi_{S}^{*}\left(D_{i}^{\beta} D_{i}^{\alpha} \psi_{S}\right)+\text { э.c. }\right\}
\end{aligned}
$$

появляются автоматически из уравнения Шредингера (через “э.с.” обозначена эрмитово сопряженная часть). Уравнение баланса импульса (24) содержит плотность магнитного момента частиц $\mathbf{M}(\mathbf{r}, t)$ :

$$
M^{\beta}(\mathbf{r}, t)=\sum_{S} \int d R \sum_{i=1}^{N} \delta\left(\mathbf{r}-\mathbf{r}_{i}\right) \mu_{\mathrm{B}} \psi_{S}^{*}\left(\hat{\sigma}_{i}^{\beta} \psi\right)_{S} .
$$

Кулоновские и спин-спиновые взаимодействия между частицами представлены в (24) слагаемыми, в которых

$$
n_{2}\left(\mathbf{r}, \mathbf{r}^{\prime}, t\right)=\sum_{S} \int d R \sum_{i, j=1}^{N} \delta\left(\mathbf{r}-\mathbf{r}_{i}\right) \delta\left(\mathbf{r}^{\prime}-\mathbf{r}_{j}\right) \psi_{S}^{*} \psi_{S}
$$

- двухчастичная плотность вероятности (нормированная на $N(N-1)$ ) нахождения двух частиц в окрестностях точек $\mathbf{r}$ и $\mathbf{r}^{\prime}$, a

$$
\begin{aligned}
M^{\alpha \beta}\left(\mathbf{r}, \mathbf{r}^{\prime}, t\right)=\sum_{S} \int d R \sum_{i, j=1}^{N} \delta\left(\mathbf{r}-\mathbf{r}_{i}\right) \delta\left(\mathbf{r}^{\prime}-\mathbf{r}_{j}\right) \mu_{\mathrm{B}}^{2} \psi_{S}^{*}\left(\hat{\sigma}_{i}^{\alpha} \hat{\sigma}_{j}^{\beta} \psi\right)_{S}= \\
=\sum_{s_{1}, \ldots, s_{N}} \int d R \sum_{i, j=1}^{N} \delta\left(\mathbf{r}-\mathbf{r}_{i}\right) \delta\left(\mathbf{r}^{\prime}-\mathbf{r}_{j}\right) \mu_{\mathrm{B}}^{2} \times \\
\quad \times \psi_{s_{1} \ldots s_{N}}^{*}(R, t) \sum_{s_{i}^{\prime}, s_{j}^{\prime}} \sigma_{s_{i} s_{i}^{\prime}}^{\alpha} \sigma_{s_{j} s_{j}^{\prime}}^{\beta} \psi_{s_{1} \ldots s_{i-1} s_{i}^{\prime} s_{i+1} \ldots s_{j-1} s_{j}^{\prime} s_{j+1} \ldots s_{N}}(R, t)
\end{aligned}
$$

- соответствуюший (27) двухчастичный тензор плотности магнитного момента.

Очевидно, что для гидродинамического описания систем со спином недостаточно уравнений баланса числа частиц и импульса. Для полного описания таких систем необходимо также уравнение динамики плотности магнитного момента $M^{\alpha}(\mathbf{r}, t)$. Это уравнение нетрудно получить путем дифференцирования выражения (27) по времени с использованием уравнения Шредингера (18) и коммутационных соотношений (20). В результате приходим к уравнению

$$
\begin{aligned}
& \frac{\partial M^{\alpha}(\mathbf{r}, t)}{\partial t}+\nabla^{\beta} J_{M}^{\alpha \beta}(\mathbf{r}, t)= \\
& \quad=\frac{e}{m c} \varepsilon^{\alpha \beta \gamma}\left\{M^{\beta}(\mathbf{r}, t) B_{\mathrm{ext}}^{\gamma}(\mathbf{r}, t)+\int d \mathbf{r}^{\prime} G^{\gamma \delta}\left(\mathbf{r}-\mathbf{r}^{\prime}\right) M^{\beta \delta}\left(\mathbf{r}, \mathbf{r}^{\prime}, t\right)\right\},
\end{aligned}
$$


где

$$
J_{M}^{\alpha \beta}(\mathbf{r}, t)=\sum_{S} \int d R \sum_{i=1}^{N} \delta\left(\mathbf{r}-\mathbf{r}_{i}\right) \frac{\mu_{\mathrm{B}}}{2 m}\left\{\psi_{S}^{*}\left(\hat{\sigma}_{i}^{\alpha} D_{i}^{\beta} \psi_{S}\right)+\left(\hat{\sigma}_{i}^{\alpha} D_{i}^{\beta} \psi_{S}\right)^{*} \psi_{S}\right\}
$$

- тензор плотности потока магнитного момента.

Уравнение (30) является обобщением известного уравнения Блоха (см., например, [10]) на случай пространственно распределенной системы частиц.

4. Поле скоростей. Для выделения потоковых скоростей в уравнениях квантовой гидродинамики необходимо представить волновую функцию в виде

$$
\psi_{S}(R, t)=\exp \left(\frac{i}{\hbar} \mathbf{v}(\mathbf{r}, t) \sum_{i=1}^{N} m \mathbf{r}_{i}\right) \varphi_{S}(\mathbf{r}, R, t)
$$

где гидродинамическая скорость $\mathbf{v}(\mathbf{r}, t)$ определяется из условия

$$
\mathbf{j}(\mathbf{r}, t)=m n(\mathbf{r}, t) \mathbf{v}(\mathbf{r}, t),
$$

при этом для нового спинора $\varphi_{S}(\mathbf{r}, R, t)$ получаем

$$
\sum_{S} \int d R \sum_{i=1}^{N} \delta\left(\mathbf{r}-\mathbf{r}_{i}\right) \frac{1}{2}\left\{\varphi_{S}^{*}\left(D_{i}^{\alpha} \varphi_{S}\right)+\left(D_{i}^{\alpha} \varphi_{S}\right)^{*} \varphi_{S}\right\}=0,
$$

$\varphi_{S}(\mathbf{r}, R, t)$ - волновая функция, определенная относительно локальной системы координат с началом в точке $\mathbf{r}$ и мгновенной скоростью $\mathbf{v}(\mathbf{r}, t)$.

Действуя производной $\mathbf{D}_{i}$ на волновую функцию $\psi(R, t)$, получаем

$$
D_{i}^{\alpha} \psi(R, t)=m v^{\alpha}(\mathbf{r}, t) \exp \left(\frac{i}{\hbar} \mathbf{v} \sum_{i=1}^{N} m \mathbf{r}_{i}\right) \varphi(\mathbf{r}, R, t)+\exp \left(\frac{i}{\hbar} \mathbf{v} \sum_{i=1}^{N} m \mathbf{r}_{i}\right) D_{i}^{\alpha} \varphi(\mathbf{r}, R, t) .
$$

В результате уравнения $(23),(24)$ и (30) приобретают вид

$$
\begin{gathered}
\frac{\partial n(\mathbf{r}, t)}{\partial t}+\nabla(n(\mathbf{r}, t) \mathbf{v}(\mathbf{r}, t))=0 \\
m n(\mathbf{r}, t)\left(\frac{\partial}{\partial t}+\mathbf{v}(\mathbf{r}, t) \nabla\right) v^{\alpha}(\mathbf{r}, t)+\nabla^{\beta} p^{\alpha \beta}(\mathbf{r}, t)=e n(\mathbf{r}, t) E_{\mathrm{ext}}^{\alpha}(\mathbf{r}, t)- \\
-\int d \mathbf{r}^{\prime} e^{2} \nabla^{\alpha} G\left(\mathbf{r}-\mathbf{r}^{\prime}\right) n_{2}\left(\mathbf{r}, \mathbf{r}^{\prime}, t\right)+\frac{e}{c} \varepsilon^{\alpha \beta \gamma} n(\mathbf{r}, t) v^{\beta}(\mathbf{r}, t) B_{\mathrm{ext}}^{\gamma}(\mathbf{r}, t)+ \\
+M^{\beta}(\mathbf{r}, t) \nabla^{\alpha} B_{\mathrm{ext}}^{\beta}(\mathbf{r}, t)+\int d \mathbf{r}^{\prime} \nabla^{\alpha} G^{\beta \gamma}\left(\mathbf{r}-\mathbf{r}^{\prime}\right) M^{\beta \gamma}\left(\mathbf{r}, \mathbf{r}^{\prime}, t\right), \\
\frac{\partial M^{\alpha}(\mathbf{r}, t)}{\partial t}+\nabla^{\beta}\left(v^{\beta}(\mathbf{r}, t) M^{\alpha}(\mathbf{r}, t)+j_{M}^{\alpha \beta}(\mathbf{r}, t)\right)= \\
=\frac{e}{m c} \varepsilon^{\alpha \beta \gamma}\left\{M^{\beta}(\mathbf{r}, t) B_{\mathrm{ext}}^{\gamma}(\mathbf{r}, t)+\int d \mathbf{r}^{\prime} G^{\gamma \delta}\left(\mathbf{r}-\mathbf{r}^{\prime}\right) M^{\beta \delta}\left(\mathbf{r}, \mathbf{r}^{\prime}, t\right)\right\},
\end{gathered}
$$

где тензоры $p^{\alpha \beta}$ и $j_{M}^{\alpha \beta}$ отличаются от соответствующих им $\Pi^{\alpha \beta}$ и $J_{M}^{\alpha \beta}$ лишь заменой спинора $\psi$ в (26) и (31) на “локальный" спинор $\varphi$. 
5. Уравнение баланса энергии. Следуя методу построения оператора плотности энергии в квантовой механике многих частиц, изложенному в работе [1], легко получить следуюшее выражение для плотности энергии (без учета внешних полей):

$$
\begin{aligned}
\varepsilon(\mathbf{r}, t)= & \int d R \sum_{i=1}^{N} \delta\left(\mathbf{r}-\mathbf{r}_{i}\right) \frac{1}{2}\left\{\psi^{+} \frac{1}{2 m}\left(D_{i}^{2} \psi\right)+\frac{1}{2 m}\left(D_{i}^{2} \psi\right)^{+} \psi\right\}+ \\
& +\int d R \sum_{i, j=1}^{N} \delta\left(\mathbf{r}-\mathbf{r}_{i}\right) \frac{1}{2} \psi^{+}\left(e^{2} G_{i j}-\mu_{\mathrm{B}}^{2} G_{i j}^{\alpha \beta} \hat{\sigma}_{i}^{\alpha} \hat{\sigma}_{j}^{\beta}\right) \psi
\end{aligned}
$$

После выделения гидродинамической скорости плотность энергии $\varepsilon(\mathbf{r}, t)$ можно представить в виде суммы конвективной части и плотности внутренней энергии $n(\mathbf{r}, t) \epsilon(\mathbf{r}, t)$ :

$$
\varepsilon(\mathbf{r}, t)=n(\mathbf{r}, t) \frac{m v^{2}(\mathbf{r}, t)}{2}+n(\mathbf{r}, t) \epsilon(\mathbf{r}, t)
$$

$\epsilon(\mathbf{r}, t)$ - удельная энергия частиц. Она определяется выражением

$$
\begin{aligned}
n(\mathbf{r}, t) \epsilon(\mathbf{r}, t)= & \int d R \sum_{i=1}^{N} \delta\left(\mathbf{r}-\mathbf{r}_{i}\right) \frac{1}{2}\left\{\varphi^{+} \frac{1}{2 m}\left(D_{i}^{2} \varphi\right)+\frac{1}{2 m}\left(D_{i}^{2} \varphi\right)^{+} \varphi\right\}+ \\
& +\frac{1}{2} \int d R \sum_{i, j=1}^{N} \delta\left(\mathbf{r}-\mathbf{r}_{i}\right) \varphi^{+}\left(e^{2} G_{i j}-\mu_{\mathrm{B}}^{2} G_{i j}^{\alpha \beta} \hat{\sigma}_{i}^{\alpha} \hat{\sigma}_{j}^{\beta}\right) \varphi .
\end{aligned}
$$

Дифференцируя соотношение (36) по времени, используя уравнение Шредингера и исключая конвективные слагаемые, получаем следуюшее уравнение баланса внутренней энергии:

$$
n\left(\frac{\partial}{\partial t}+\mathbf{v} \nabla\right) \epsilon+p^{\alpha \beta} \nabla^{\alpha} v^{\beta}+\nabla \mathbf{q}=-\left(\nabla^{\alpha} B_{\mathrm{ext}}^{\beta}\right) j_{M}^{\alpha \beta}-\mathfrak{W}
$$

где

$$
\begin{aligned}
\mathbf{q}(\mathbf{r}, t)= & \int d R \sum_{i=1}^{N} \delta\left(\mathbf{r}-\mathbf{r}_{i}\right) \frac{1}{8 m^{2}}\left\{\varphi^{+}\left(\mathbf{D}_{i} \mathbf{D}_{i}^{2} \varphi\right)+\left(\mathbf{D}_{i} \varphi\right)^{+}\left(\mathbf{D}_{i}^{2} \varphi\right)+\text { к.c. }\right\}+ \\
& +\frac{1}{2} \int d R \sum_{i, j=1}^{N} \delta\left(\mathbf{r}-\mathbf{r}_{i}\right) \frac{1}{2 m}\left\{\varphi^{+}\left(e^{2} G_{i j}-\mu_{\mathrm{B}}^{2} G_{i j}^{\alpha \beta} \hat{\sigma}_{i}^{\alpha} \hat{\sigma}_{j}^{\beta}\right)\left(\mathbf{D}_{i} \varphi\right)+\text { k.c. }\right\}
\end{aligned}
$$

- плотность теплового потока внутренней энергии.

Плотность работы $\mathfrak{W}(\mathbf{r}, t)$, совершаемой системой со спинами на тепловых скоростях (относительно локальной системы координат, связанной с гидродинамическим потоком) во внешнем магнитном поле, имеет вид

$$
\begin{aligned}
\mathfrak{W}(\mathbf{r}, t)= & \frac{1}{2} \int d \mathbf{r}^{\prime}\left(v^{\beta}\left(\mathbf{r}^{\prime}, t\right)-v^{\beta}(\mathbf{r}, t)\right) \times \\
& \times\left\{n_{2}\left(\mathbf{r}, \mathbf{r}^{\prime}, t\right) e^{2} \nabla^{\beta} G\left(\mathbf{r}-\mathbf{r}^{\prime}\right)-M^{\alpha \gamma}\left(\mathbf{r}, \mathbf{r}^{\prime}, t\right) \nabla^{\beta} G^{\alpha \gamma}\left(\mathbf{r}-\mathbf{r}^{\prime}\right)\right\}-
\end{aligned}
$$




$$
\begin{aligned}
& -\frac{1}{2} \int d \mathbf{r}^{\prime}\left(\mathfrak{M}^{\alpha \gamma \beta}\left(\mathbf{r}, \mathbf{r}^{\prime}, t\right)+\mathfrak{M}^{\alpha \gamma \beta}\left(\mathbf{r}^{\prime}, \mathbf{r}, t\right)\right) \nabla^{\beta} G^{\alpha \gamma}\left(\mathbf{r}-\mathbf{r}^{\prime}\right)- \\
& -\frac{e}{m c} B_{\mathrm{ext}}^{\alpha}(\mathbf{r}, t) \varepsilon^{\alpha \beta \gamma} \int d \mathbf{r}^{\prime} M^{\beta \delta}\left(\mathbf{r}, \mathbf{r}^{\prime}, t\right) G^{\gamma \delta}\left(\mathbf{r}-\mathbf{r}^{\prime}\right),
\end{aligned}
$$

где тензор 3-го ранга $\mathfrak{M}^{\alpha \gamma \beta}\left(\mathbf{r}, \mathbf{r}^{\prime}, t\right)$ имеет смысл двухчастичной плотности потока магнитного момента относительно локальной системы координат (на тепловых скоростях):

$$
\mathfrak{M}^{\alpha \gamma \beta}\left(\mathbf{r}, \mathbf{r}^{\prime}, t\right)=\int d R \sum_{i, j=1}^{N} \delta\left(\mathbf{r}-\mathbf{r}_{i}\right) \delta\left(\mathbf{r}^{\prime}-\mathbf{r}_{j}\right) \frac{\mu_{\mathrm{B}}^{2}}{2 m}\left\{\varphi^{+} \hat{\sigma}_{i}^{\alpha} \hat{\sigma}_{j}^{\gamma}\left(D_{i}^{\beta} \varphi\right)+\left(D_{i}^{\beta} \varphi\right)^{+} \hat{\sigma}_{i}^{\alpha} \hat{\sigma}_{j}^{\gamma} \varphi\right\}
$$

Заметим, что тензор $\mathfrak{M}^{\alpha \gamma \beta}$ является симметричным по первым двум индексам, так как входящие в его определение матрицы Паули относятся к разным частицам и, следовательно, коммутируют.

Интегро-дифференциальное уравнение (37) представляет собой исходное уравнение неравновесной квантовой термодинамики системы заряженных частиц со спином.

Полученная вьше система уравнений квантовой гидродинамики $(33),(34),(37)$ и (38) является точной в том смысле, что при ее выводе не привлекались какие-либо дополнительные для квантовой механики предположения. Однако эти уравнения являются незамкнутыми, так как содержат в слагаемых с взаимодействием двухчастичные функции $n_{2}\left(\mathbf{r}, \mathbf{r}^{\prime}, t\right), M^{\alpha \beta}\left(\mathbf{r}, \mathbf{r}^{\prime}, t\right)$ и $\mathfrak{M}^{\alpha \gamma \beta}\left(\mathbf{r}, \mathbf{r}^{\prime}, t\right)$, которые, в свою очередь, не выражаются через одночастичные. Для их определения можно получить соответствующие уравнения, которые, как естественно ожидать, будут содержать трехчастичные функции. Продолжая эту процедуру, мы придем к цепочке уравнений для физических полей различной тензорной размерности, являющейся гидродинамическим аналогом цепочки Боголюбова. Такая цепочка уравнений полностью эквивалентна уравнению Шредингера. Кроме того, приведенные выше уравнения, как и уравнения классической гидродинамики, должны быть дополнены уравнениями состояния, конкретизирующими как состояние системы частиц, так и характер физических процессов в такой системе.

Для решения конкретных физических задач необходимо оборвать эту цепочку, тем самым произведя процедуру замыкания системы уравнений.

6. Проблема замыкания уравнений баланса и одночастичное уравнение Шредингера. Двухчастичные гидродинамические функции являются функционалами волновой функции $\varphi(\mathbf{r}, R, t)$. Процедура получения замкнутого аппарата означает нахождение функциональной зависимости двухчастичных функций от одночастичных полевых функций $n(\mathbf{r}, t), \mathbf{v}(\mathbf{r}, t), \mathbf{M}(\mathbf{r}, t)$ и $\epsilon(\mathbf{r}, t)$.

Для установления такой зависимости выделим в волновой функции в каждый момент времени антисимметризованное произведение одночастичных ортогональных спиноров $\varphi_{f_{i}}\left(x_{i}, t\right)$ :

$$
\varphi\left(\mathbf{r}, x_{1}, \ldots, x_{N}, t\right)=\left\langle x_{1}, \ldots, x_{N} \mid n_{1}, n_{2}, \ldots ; t\right\rangle+\zeta\left(\mathbf{r}, x_{1}, \ldots, x_{N}, t\right),
$$

6 Теоретическая и математическая физика, т. 126, № 1, 2001 г. 
где

$$
\left\langle x_{1}, \ldots, x_{N} \mid n_{1}, n_{2}, \ldots ; t\right\rangle=\frac{1}{N !} \sum_{P}(-1)^{P} P\left(\varphi_{f_{1}}\left(x_{1}, t\right) \ldots \varphi_{f_{N}}\left(x_{N}, t\right)\right) .
$$

Через $x_{i}$ мы для краткости обозначили совокупность пространственных координат $\mathbf{r}_{i}$ и спинорного индекса $s_{i}$. Поскольку одночастичные спиноры $\varphi_{f_{i}}\left(x_{i}, t\right)$ не зависят от координат в физическом пространстве $\mathbf{r}$, то зависимость от $\mathbf{r}$ “локальной” волновой функции $\varphi(\mathbf{r}, R, t)$ может реализовываться через числа заполнения $n_{f}$, т.е. числа заполнения являются функциями $\mathbf{r}$ и в обшем случае $t$.

Потребуем, чтобы одночастичные волновые функции $\varphi_{f_{i}}\left(x_{i}, t\right)$ хорошо аппроксимировали гидродинамические функции, такие как концентрация, ток, плотность магнитного момента и т.д. Что касается возникающих при этом дополнительных условий на функцию $\zeta$, то здесь ситуация сходна с методом Чепмена-Энскога, в котором плотность числа частиц, поле скоростей и плотность кинетической энергии определяются интегрированием только локально-равновесной части функции распределения. Соответствующие интегралы от неравновесной составляюшей предполагаются равными нулю. Аналогичные предположения содержит также метод Крылова-Боголюбова: высшие гармоники не дают вклада в полную амплитуду нелинейных колебаний.

Эволюция одночастичного спинора $\varphi_{f_{i}}\left(x_{i}, t\right)$ описывается одночастичным уравнением Шредингера

$$
i \hbar \frac{\partial \varphi_{f}(x, t)}{\partial t}=\left(\hat{h} \varphi_{f}\right)(x, t)
$$

с эффективным одночастичным гамильтонианом $\hat{h}$. Потребуем, чтобы одночастичный гамильтониан $\hat{h}$ был согласованным с уравнениями $(33)-(35),(37),(38)$ и уравнениями поля. При этом основными для нас уравнениями, определяюшими динамику системы, являются уравнения гидродинамики. Уравнение (40) носит вспомогательный характер. Поэтому нет необходимости требовать, чтобы одночастичный гамильтониан $\hat{h}$ содержал квантовые корреляции и уравнение (40) было полностью эквивалентно уравнениям квантовой гидродинамики.

Подстановка (39) в двухчастичные функции (28), (29) дает следуюшее групповое разложение:

$$
\begin{aligned}
n_{2}\left(\mathbf{r}, \mathbf{r}^{\prime}, t\right)= & n(\mathbf{r}, t) n\left(\mathbf{r}^{\prime}, t\right)+g\left(\mathbf{r}, \mathbf{r}^{\prime}, t\right)+\tilde{g}[\zeta], \\
M^{\alpha \beta}\left(\mathbf{r}, \mathbf{r}^{\prime}, t\right)= & M^{\alpha}(\mathbf{r}, t) M^{\beta}\left(\mathbf{r}^{\prime}, t\right)+\mathfrak{m}^{\alpha \beta}\left(\mathbf{r}, \mathbf{r}^{\prime}, t\right)+\widetilde{\mathfrak{m}}^{\alpha \beta}[\zeta], \\
\mathfrak{M}^{\alpha \gamma \beta}\left(\mathbf{r}, \mathbf{r}^{\prime}, t\right)= & \frac{1}{2}\left\{j_{M}^{\alpha \beta}(\mathbf{r}, t) M^{\gamma}\left(\mathbf{r}^{\prime}, t\right)+j_{M}^{\gamma \beta}(\mathbf{r}, t) M^{\alpha}\left(\mathbf{r}^{\prime}, t\right)\right\}+ \\
& +\mathfrak{h}^{\alpha \gamma \beta}\left(\mathbf{r}, \mathbf{r}^{\prime}, t\right)+\tilde{\mathfrak{h}}^{\alpha \gamma \beta}[\zeta],
\end{aligned}
$$

где $g\left(\mathbf{r}, \mathbf{r}^{\prime}, t\right), \mathfrak{m}^{\alpha \beta}\left(\mathbf{r}, \mathbf{r}^{\prime}, t\right)$ и $\mathfrak{h}^{\alpha \gamma \beta}\left(\mathbf{r}, \mathbf{r}^{\prime}, t\right)$ определяют обменные корреляции, а $\tilde{g}[\zeta]$, $\tilde{\mathfrak{h}}^{\alpha \gamma \beta}[\zeta]$ и $\widetilde{\mathfrak{m}}^{\alpha \beta}[\zeta]$ - слагаемые, содержащие функцию $\zeta\left(x_{1}, \ldots, x_{N}, t\right)$. Подстановка (41) в уравнения (33)-(35), (37) и (38) приводит к появлению самосогласованных электрического $\mathbf{E}_{\text {int }}$ и магнитного $\mathbf{B}_{\text {spin }}$ полей, удовлетворяющих следующим уравнениям:

$$
\begin{gathered}
\operatorname{div} \mathbf{E}_{\text {int }}=4 \pi e n, \quad \operatorname{div} \mathbf{B}_{\text {spin }}=0, \\
\operatorname{rot}\left(\mathbf{B}_{\text {spin }}-4 \pi \mathbf{M}\right)=0, \quad \operatorname{rot} \mathbf{E}_{\text {int }}=0 .
\end{gathered}
$$


Уравнения (34), (35) не содержат магнитного поля, вызванного диамагнитными токами. Кроме того, в выражении для силы Лоренца отсутствует взаимодействие тока с полем $\mathbf{B}_{\text {spin. }}$. Эти обстоятельства связаны с недостатком исходного нерелятивистского гамильтониана, взятого за основу для получения гидродинамических уравнений. Указанный недостаток может быть устранен, в частности, путем сравнения уравнений классической и квантовой гидродинамики. Классические уравнения для частиц с электромагнитным взаимодействием содержат аддитивно внешнее магнитное поле и поле, обусловленное токами. Соответствуюшие квантовые уравнения в пределе должны совпадать с классическими и, следовательно, содержать информацию о всей совокупности гидродинамических эффектов классической физики. Это возможно, если в уравнениях квантовой гидродинамики присутствует полная сила Лоренца и учтено взаимодействие магнитного момента с полным магнитным полем. Таким образом, учитывая требования принципа соответствия непосредственно в уравнениях баланса, мы оставляем без внимания проблему конструирования нового гамильтониана на основании того же принципа и приходим к уравнениям

$$
\begin{aligned}
& m n(\mathbf{r}, t)\left(\frac{\partial}{\partial t}+\mathbf{v}(\mathbf{r}, t) \nabla\right) v^{\alpha}(\mathbf{r}, t)+\nabla^{\beta} p^{\alpha \beta}(\mathbf{r}, t)= \\
& =e n(\mathbf{r}, t)\left(E^{\alpha}(\mathbf{r}, t)+\frac{1}{c} \varepsilon^{\alpha \beta \gamma} v^{\beta}(\mathbf{r}, t) B^{\gamma}(\mathbf{r}, t)\right)+ \\
& +M^{\beta}(\mathbf{r}, t) \nabla^{\alpha} B^{\beta}(\mathbf{r}, t)-\int d \mathbf{r}^{\prime} e^{2} \nabla^{\alpha} G\left(\mathbf{r}-\mathbf{r}^{\prime}\right) g\left(\mathbf{r}, \mathbf{r}^{\prime}, t\right)+ \\
& +\int d \mathbf{r}^{\prime} \nabla^{\alpha} G^{\beta \gamma}\left(\mathbf{r}-\mathbf{r}^{\prime}\right) \mathfrak{m}^{\beta \gamma}\left(\mathbf{r}, \mathbf{r}^{\prime}, t\right)+f^{\alpha}[\zeta], \\
& \frac{\partial M^{\alpha}(\mathbf{r}, t)}{\partial t}+\nabla^{\beta}\left(v^{\beta}(\mathbf{r}, t) M^{\alpha}(\mathbf{r}, t)+j_{M}^{\alpha \beta}(\mathbf{r}, t)\right)= \\
& =\frac{e}{m c} \varepsilon^{\alpha \beta \gamma} M^{\beta}(\mathbf{r}, t) B^{\gamma}(\mathbf{r}, t)+ \\
& +\frac{e}{m c} \varepsilon^{\alpha \beta \gamma} \int d \mathbf{r}^{\prime} G^{\gamma \delta}\left(\mathbf{r}-\mathbf{r}^{\prime}\right) \mathfrak{m}^{\beta \delta}\left(\mathbf{r}, \mathbf{r}^{\prime}, t\right)+f_{M}^{\alpha}[\zeta],
\end{aligned}
$$

где $f^{\alpha}[\zeta]$ и $f_{M}^{\alpha}[\zeta]$ - слагаемые, содержашие функцию $\zeta\left(x_{1}, \ldots, x_{N}, t\right)$, а поля $\mathbf{E}(\mathbf{r}, t)$ и $\mathbf{B}(\mathbf{r}, t)$ удовлетворяют полным уравнениям Максвелла:

$$
\begin{gathered}
\operatorname{div} \mathbf{E}=4 \pi e n, \quad \operatorname{div} \mathbf{B}=0 \\
\operatorname{rot}(\mathbf{B}-4 \pi \mathbf{M})=\frac{1}{c} \frac{\partial \mathbf{E}}{\partial t}+\frac{4 \pi}{c} e n \mathbf{v}, \quad \operatorname{rot} \mathbf{E}=-\frac{1}{c} \frac{\partial \mathbf{B}}{\partial t} .
\end{gathered}
$$

В качестве одночастичного гамильтониана естественно взять гамильтониан с самосогласованным полем:

$$
i \hbar \frac{\partial \varphi_{f}}{\partial t}=\frac{1}{2 m}\left(\widehat{\mathbf{p}}-\frac{e}{c} \mathbf{A}(\mathbf{r}, t)\right)^{2} \varphi_{f}-\mu_{\mathrm{B}}(\hat{\boldsymbol{\sigma}} \mathbf{B}(\mathbf{r}, t)) \varphi_{f}+e \Phi(\mathbf{r}, t) \varphi_{f} .
$$

Скалярньй $\Phi(\mathbf{r}, t)$ и векторный $\mathbf{A}(\mathbf{r}, t)$ потенциалы электромагнитного поля являются решениями одновременно уравнений Максвелла (44) с источниками $n(\mathbf{r}, t), \mathbf{v}(\mathbf{r}, t)$ 
и $\mathbf{M}(\mathbf{r}, t)$ и уравнений квантовой гидродинамики (42) и (43) с обменными корреляционными функциями $g\left(\mathbf{r}, \mathbf{r}^{\prime}, t\right), \mathfrak{m}^{\alpha \beta}\left(\mathbf{r}, \mathbf{r}^{\prime}, t\right)$ и $\mathfrak{h}^{\alpha \gamma \beta}\left(\mathbf{r}, \mathbf{r}^{\prime}, t\right)$, функционально связанными с одночастичными волновыми функциями $\varphi_{f}(\mathbf{r}, s, t)$. Таким образом, задача становится замкнутой и самосогласованной не только относительно полей $\Phi(\mathbf{r}, t)$ и $\mathbf{A}(\mathbf{r}, t)$, но и относительно одночастичных спиноров $\varphi_{f}(\mathbf{r}, t)$. Заметим, что рассмотрение процессов при конечных температурах приводит к необходимости включения в рассматриваемую систему уравнений также уравнения баланса энергии (37) и (38).

Общая схема получения замкнутого аппарата квантовой гидродинамики после расчета корреляционных функций реализована в части II настоящей работы для нестационарной задачи о многоэлектронном атоме.

\section{Список литературы}

[1] Л. С. Кузьменков, С.Г. Максимов. ТМФ. 1999. Т. 118. С. 287-304.

[2] К. П. Гуров. Основания кинетической теории. М.: Наука, 1966.

[3] Н. Н. Боголюбов. Избранные труды. Т. 3. Киев: Наукова думка, 1971.

[4] А.И. Ахиезер, В. Г. Барьяхтар, С. В. Пелетминский. Спиновые волны. М.: Наука, 1967.

[5] О. Бор, Б. Моттельсон. Структура атомного ядра. Т. 1. М.: Мир, 1971.

[6] N.W. Ashcroft, N. D. Mermin. Solid State Physics. New York: Holt, Rinehart and Winston, 1976.

[7] В. Б. Берестецкий, Е. М. Лифииц, Л. П. Питаевский. Квантовая электродинамика. М.: Наука, 1989.

[8] И. В. Александров. Теория ядерного магнитного резонанса. М.: Наука, 1964.

[9] В. С. Владимиров. Обобщенные функции в математической физике. М.: Наука, 1979.

[10] R. K. Wangsness. Phys. Rev. 1955. V. 98. № 4. P. 927.

Поступила в редакцию 22.VI.2000 г. 